\title{
Effect of the Order-Disorder Transition on the Seebeck Coefficient of Nanostructured Thermoelectric $\mathrm{Cu}_{2} \mathrm{ZnSnS}_{4}$
}

\author{
Eleonora Isotta ${ }^{1,2} \mathbb{D}$, Carlo Fanciulli ${ }^{3}$, Nicola M. Pugno ${ }^{1,2,4,5}$ and Paolo Scardi ${ }^{1, *(1)}$ \\ 1 Department of Civil, Environmental and Mechanical Engineering, University of Trento, via Mesiano 77, \\ 38123 Trento, Italy; eleonora.isotta@unitn.it (E.I.); nicola.pugno@unitn.it (N.M.P.) \\ 2 Laboratory of Bio-Inspired and Graphene Nanomechanics, Department of Civil, Environmental and \\ Mechanical Engineering, University of Trento, via Mesiano 77, 38123 Trento, Italy \\ 3 National Research Council of Italy-Institute of Condensed Matter Chemistry and Technologies for \\ Energy (CNR-ICMATE), Lecco Unit, via Previati 1/E, 23900 Lecco, Italy; carlo.fanciulli@cnr.it \\ 4 Ket-Lab, Edoardo Amaldi Foundation, Via del Politecnico snc, 00133 Rome, Italy \\ 5 School of Engineering and Materials Science, Queen Mary University of London, Mile End Road, \\ London E1 4NS, UK \\ * Correspondence: paolo.scardi@unitn.it
}

Received: 23 April 2019; Accepted: 13 May 2019; Published: 17 May 2019

\begin{abstract}
Bulk samples of kesterite $\left(\mathrm{Cu}_{2} \mathrm{ZnSnS}_{4}, \mathrm{CZTS}\right)$ were produced by cold-pressing and sintering of CZTS powders obtained via reactive ball-milling. An increase in the Seebeck coefficient of more than $100 \mu \mathrm{V} / \mathrm{K}$, almost doubling the expected value, is noticed around a temperature of $260{ }^{\circ} \mathrm{C}$. As pointed out by thermal analyses, this is due to a second order transition of kesterite from an ordered I-4 to a disordered I-42m crystal structure. Conversely to what happens for solar cell materials, where the transition is considered to be detrimental for the performance, it appears to be beneficial for the thermoelectric Seebeck coefficient, suggesting that higher crystal symmetry and cation-disorder due to the transition lead to thermopower enhancement.
\end{abstract}

Keywords: order-disorder transition; Seebeck coefficient; kesterite; CZTS; nanostructured materials; thermoelectric materials; crystal structure symmetry

\section{Introduction}

Thermoelectric materials can operate the conversion of a heat gradient into a voltage drop through the Seebeck effect [1,2]. The performance is usually expressed through the figure of merit $z T=S^{2} T /(\rho k)$, where $S, \rho, T$, and $k$ are, respectively, the Seebeck coefficient, electrical resistivity, absolute temperature, and thermal conductivity [3] although recently the thermoelectric quality factor $\beta$ has been proposed, as it draws attention to the most fundamental material parameters to be improved $\left(\beta \propto N_{v} /\left(m_{I}^{*} k_{L}\right)\right.$, with $N_{v}$, band degeneracy, $m_{I}^{*}$, inertial effective mass along the conduction direction, and $k_{L}$, lattice thermal conductivity) $[4,5]$. Kesterite $\left(\mathrm{Cu}_{2} \mathrm{ZnSnS}_{4}, \mathrm{CZTS}\right)$ is a direct band gap p-type semiconductor that has recently been studied as a thermoelectric material [6-10]. The interest arises from the low cost, easy availability and non-toxicity, in addition to its low thermal conductivity (from $2.95 \mathrm{~W} /(\mathrm{m} \mathrm{K}$ ) at $300 \mathrm{~K}$ to $0.97 \mathrm{~W} /(\mathrm{m} \mathrm{K})$ at $700 \mathrm{~K}$ [7]). The reported values of figure of merit $z T$ for kesterite remain quite low, ranging from $4.6 \times 10^{-5}$ at $300 \mathrm{~K}$ to 0.026 at $700 \mathrm{~K}$ [7]. For this reason several studies have focused on doping to increase the electrical conductivity [6,7], without considering, until now, the importance of structural aspects and the role of phase transformations. Kesterite is also an object of intense research for the realization of absorbing layers in thin-film solar cells, valued for the high absorbance and nearly optimal band gap $(\sim 1.5 \mathrm{eV})$, which make it a candidate for the substitution of 
rare and potentially toxic metal chalcogenides [11-13]. In its usual form, kesterite has a tetragonal crystal structure belonging to space group I-4. Quite recently, it has been reported that a reversible second-order phase transition occurs at a critical temperature of $260 \pm 10{ }^{\circ} \mathrm{C}[14,15]$. This phase transition appears to involve the $\mathrm{Cu}-\mathrm{Zn}$ planes in the crystal structure. As depicted in Figure 1, below the critical temperature $\mathrm{Cu}$ and $\mathrm{Zn}$ are in their ordered configuration, occupying respectively the $2 c$ and $2 d$ Wyckoff positions. Above the critical temperature, $\mathrm{Cu}$ and $\mathrm{Zn}$ are randomly distributed in the planes, leading to a transition to a $I-42 m$ crystal structure where both cations occupy $4 d$ Wyckoff positions. This order-disorder transition has been linked to the low formation energy of the Cu-Zn antisite defects [16]. At room temperature, kesterite has been reported to show a higher order degree, although this is primarily related to the thermal history of the sample and to its stoichiometry [17], and complete order is theoretically possible only at $0 \mathrm{~K}$ [15]. In photovoltaic applications, disorder has been reported to mainly cause a decrease in the bandgap energy Eg, leading to a lower open circuit voltage [15], but also a higher short-circuit current density [18,19]. Since $\mathrm{Zn}^{+2}$ and $\mathrm{Cu}^{+1}$ are isoelectronic, it is difficult to observe and quantify disorder with conventional X-Ray Diffraction (XRD), where the phase transition is just noticed as a smooth change of cell parameter with the temperature [20,21]. Other techniques, however, proved more effective such as solid-state nuclear magnetic resonance [22], UV-Vis spectroscopy for bandgap measurement [18,23], Raman spectroscopy [15,22,24], neutron scattering [21], and resonant XRD [25]. In this work we show how to effectively observe and study the order-disorder transition with the measurement of Seebeck coefficient, and we confirm the results with the aid of accurate thermal analyses.

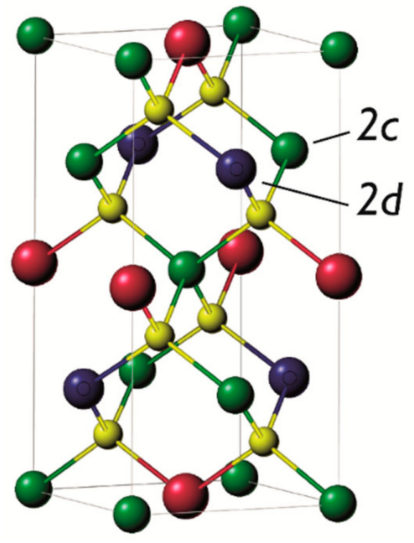

a

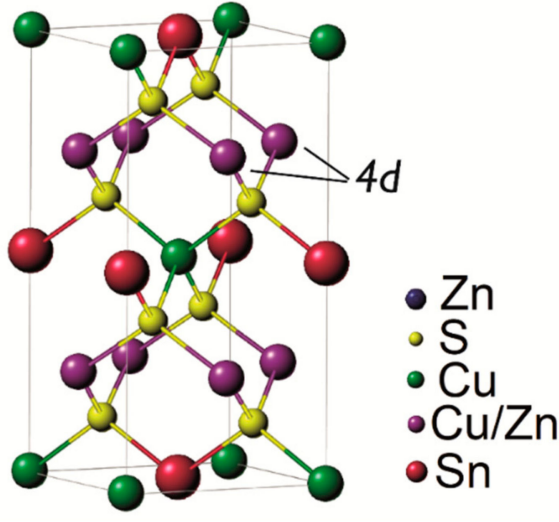

b

Figure 1. Ordered $I-4$ (a) and disordered $I-42 m(\mathbf{b})$ crystal structures of kesterite. In the ordered state $\mathrm{Cu}$ and $\mathrm{Zn}$ occupy respectively $2 c$ and $2 d$ Wyckoff positions while above the transition temperature, they appear as randomly distributed in the planes of $4 d$ Wyckoff positions $[14,15,26]$.

Nanostructured kesterite is usually produced by chemical synthesis $[27,28]$. This approach typically requires a thermal treatment well above the critical temperature, and a slow cooling down to obtain, to the extent possible, defect-free and ordered crystals, deemed appropriate for the performance of thin film solar cells [14]. However, the effect of a production route of CZTS powders based on reactive milling at room temperature of precursors such as elementary $(\mathrm{Cu}, \mathrm{Zn}, \mathrm{Sn})$ metals and sulfur is not well known. This is the approach followed in the present work to obtain a nanostructured material with finely dispersed and intrinsically disordered crystalline domains, designed to preserve the nanostructure after sintering and in operating conditions. Furthermore, we show the effect of the order-disorder transition, which is known for photovoltaic kesterite, but has not yet been investigated as regards the effect on thermoelectric properties. 


\section{Materials and Methods}

Kesterite powders have been produced from stoichiometric precursors ( $\mathrm{Cu}$ powder, $<75 \mu \mathrm{m}$, 99\%; Zn powder, purum, 99\%; Sn powder, puriss, 99\%; S flakes, purum, 99.5\%; all by SigmaAldrich (Saint Louis, MO, USA) via reactive ball-milling with brass balls and vials in a planetary mill (Fritsch P4 Pulverisette 4, Idar-Oberstein, Germany) adding $150 \mu \mathrm{L}$ of ethanol (99.8\%, SigmaAldrich) as lubricant [29]. Milling has been performed for $60 \mathrm{~min}$ with jar rotation $\omega=-540 \mathrm{rpm}$ and main disk revolution $\Omega=300 \mathrm{rpm}$ [30]. Powders have been cold-pressed with a manual pressing machine and a pressure of $25.5 \mathrm{MPa}$ to obtain disks with diameter around $20 \mathrm{~mm}$ and thickness around $1.5 \mathrm{~mm}$. The final disks have then been sintered in a tubular oven in Ar flux for $60 \mathrm{~min}$ at $300{ }^{\circ} \mathrm{C}$ (heating rate $\mathrm{r}=20 \mathrm{~K} / \mathrm{min}$ ) followed by $20 \mathrm{~min}$ at $560{ }^{\circ} \mathrm{C}\left(\mathrm{r}=20 \mathrm{~K} / \mathrm{min}\right.$ up to $520{ }^{\circ} \mathrm{C}$ and $10 \mathrm{~K} / \mathrm{min}$ from 520 to $560^{\circ} \mathrm{C}$ ) [31]. Some of the disks have been naturally cooled down to room temperature inside the furnace atmosphere, for a total cooling time of $8 \mathrm{~h}$, others have been quenched in air to room temperature. XRD (Rigaku PMG, Tokyo, Japan, Cu K $\alpha$ radiation), Trasmission Electron Microscopy (TEM) imaging, Selected Area Electron Diffraction (SAED) and high-magnification Energy Dispersive X-ray Analysis (EDXS) (HR-S/TEM ThermoFischer TALOS 200 s, Thermo Fischer Scientific, Waltham, MA, USA) have been used to characterize the samples. Thermal analyses and specific heat measurements have been performed with a Thermal Analysis Q100 DSC instrument (TA Instruments, New Castle, DE, USA). This instrument allows a modulated mode (MDSC) for calorimetric analyses that is useful for studying materials with low thermal conductivity. The modulated mode has been used in two ways. In isothermal measurements, the temperature modulation has been applied to the thermal equilibrated sample to determine the specific heat as a function of temperature. In ramp mode, the temperature modulation has been applied to the imposed ramp rate $(2 \mathrm{~K} / \mathrm{min})$, allowing the identification of the reversible and non-reversible contributions to the heat flow signal from the sample. In all cases, the applied modulation has a period $\mathrm{t}=120 \mathrm{~s}$, and semi-amplitude $\mathrm{A}=0.5^{\circ} \mathrm{C}$. Absolute Seebeck coefficient (platinum standard) has been measured in a 4-contact configuration with Linseis LZT-Meter (Linseis Messgeraete $\mathrm{GmbH}$, Selb, Germany) from $50{ }^{\circ} \mathrm{C}$ to $450{ }^{\circ} \mathrm{C}$, with step of $10^{\circ} \mathrm{C}$, a heating rate of $10 \mathrm{~K} / \mathrm{min}$ and an effective temperature gradient of approximately $10^{\circ} \mathrm{C}$.

\section{Results and Discussion}

XRD data for both slowly cooled and quenched samples are shown in Figure 2, together with result of a modeling made with TOPAS 7 [32], whereas TEM and SAED images for the slowly cooled tablet are visible in Figure 3.

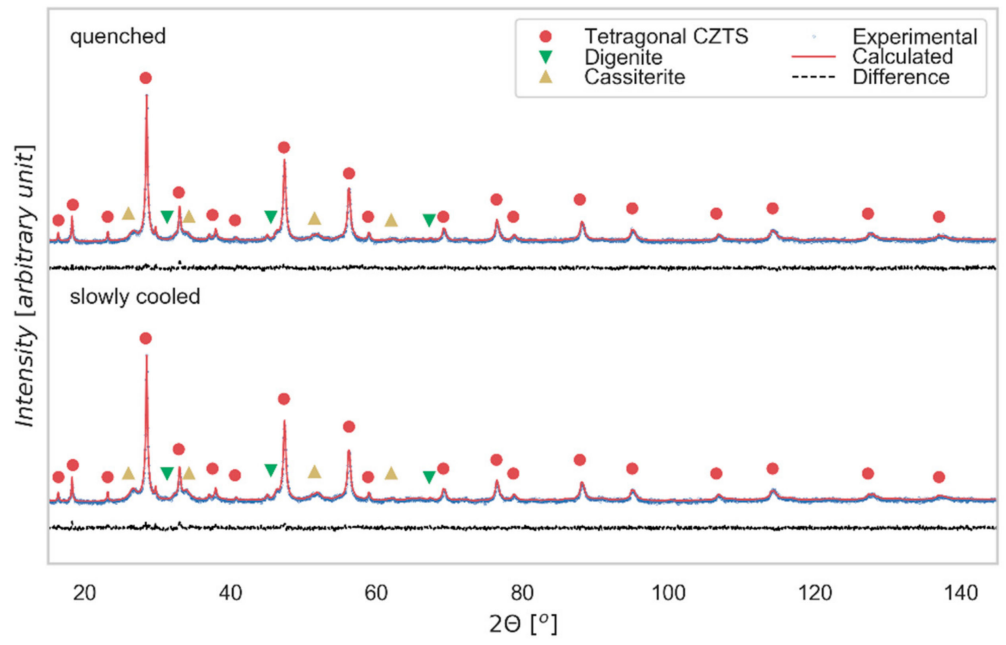

Figure 2. X-Ray Diffraction patterns. XRD data Rietveld refinement (performed with TOPAS 7) and phase identification for the slowly cooled and quenched samples. The difference between experimental data (blue) and model (red) is shown below (residual, black line). 


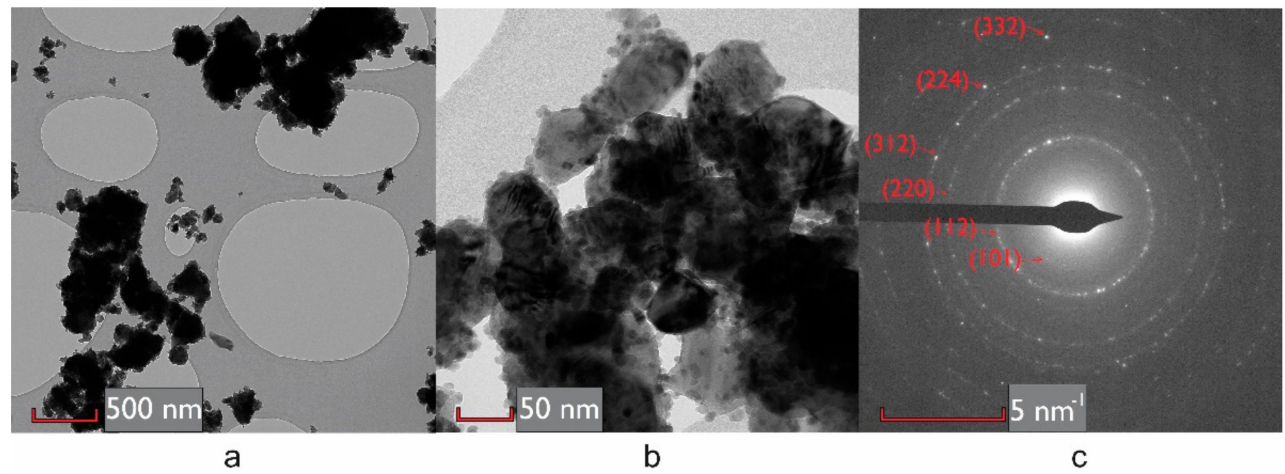

Figure 3. TEM and SAED images: $(\mathbf{a}, \mathbf{b})$ TEM images of slowly cooled sample ground to powder; (c) SAED pattern of the area shown in Figure 3a, Miller indices of scattering planes corresponding to a tetragonal crystal structure are indicated.

The XRD pattern shows the expected reflections of tetragonal kesterite including the distinctive weak peaks at $2 \theta \sim 18^{\circ}, 23^{\circ}, 38^{\circ}$, which reveal the main difference with respect to the cubic sphalerite-type structure [31]. The same indexing is confirmed by SAED (Figure 3c), where it is possible to notice the diffraction ring corresponding to the (101) plane, distinctive to the tetragonal structure. For both cooling processes, kesterite is the main phase. As expected for the isoelectronic character of $\mathrm{Cu}^{+1}$ and $\mathrm{Zn}^{+2}, \mathrm{XRD}$ cannot capture any relevant difference in the pattern of the two samples [15]. Additional peaks refer to secondary phases, identified by a search-match procedure supported by the PDF-4+ database (ICDD, 2019 [33]), and then quantified by the modeling of the XRD patterns as cassiterite $\mathrm{SnO}_{2}$ (weight fraction 12(1) \% for slowly cooled sample and 9(1) \% for quenched sample) and digenite $\mathrm{Cu}_{7,2} \mathrm{~S}_{4}$ (weight fraction 5(1) \% in both samples). The last phase might have a positive role, as it has been observed that its partially-metallic nature contributes to improving the electrical conductivity of CZTS thin films [8].

TEM images (Figure 3) show kesterite in grains and agglomerates ranging from about $10 \mathrm{~nm}$ to over $300 \mathrm{~nm}$. Cassiterite, identified by EDXS, appears in small nucleated domains of around $10 \mathrm{~nm}$ preferentially located at grain boundaries of CZTS (Figure 3b). EDXS on single grains shows stoichiometry fluctuations from grain to grain of the kesterite phase. From these observations we noticed a tendency towards bimodality in the size distribution of kesterite: two fractions are mainly identified, a bigger one, in the range of hundreds of nanometers and a smaller one, in the range of tens of nanometers (see Figure 3a). This is consequence of the mild sintering process, designed to preserve the nanostructure in bulk samples, made for short time at $560{ }^{\circ} \mathrm{C}(833 \mathrm{~K})$, well below the melting point of $1260 \mathrm{~K}$ [34]. Moreover, the high dispersion of domain sizes in the starting powder made by ball milling causes differences in grain aggregation and packing during the preparation of the green. In fact, the density of the starting pallets after cold pressing was $3.4 \mathrm{~g} / \mathrm{cm}^{3}$ (geometrically measured on disk samples), $75 \%$ of the theoretical density reported for CZTS $\left(4.56 \mathrm{~g} / \mathrm{cm}^{3}\right.$ [35]). Density fluctuations inevitably lead to differences in the growth rate of grains, resulting in the final inhomogeneous grain size distribution. Based on the observed bimodality, we used two different-size kesterite fractions to model XRD data. A TOPAS macro based on Whole Powder Pattern Modeling (WPPM) (Scardi, 2002 [36]) has been used to refine the domain size in terms of lognormal distributions of spherical domains [37]. For both samples, the estimated mean domain sizes are 20(2) $\mathrm{nm}$ for the smaller fraction (45(2)\% of total CZTS) and 180(15) $\mathrm{nm}$ for the bigger fraction (55(2)\%), matching the high dispersion of dimension that was experimentally observed with TEM analyses. The micro strain is quantified as $0.0004(1)$ and 0.0017(2), respectively for larger and smaller fractions. We believe that this should be considered, at least partially, as an effect of the stoichiometry fluctuation, confirmed by EDXS, rather than strain. In fact, fluctuations in the composition may cause the cell parameter to slightly vary, thus having multiple peak positions for the same reflection that lead to strain-like peak-broadening [38]. 
Figure 4a shows the absolute Seebeck coefficient measured on the slowly cooled sample on heating and cooling temperature ramp. Values are positive, confirming kesterite p-type behavior and range between $40 \mu \mathrm{V} / \mathrm{K}$ at $50{ }^{\circ} \mathrm{C}$ and $315 \mu \mathrm{V} / \mathrm{K}$ at $450{ }^{\circ} \mathrm{C}$. In this temperature range, it is interesting to note the marked increase of Seebeck coefficient occurring between $210{ }^{\circ} \mathrm{C}$ and $290{ }^{\circ} \mathrm{C}$, where values pass from around $80 \mu \mathrm{V} / \mathrm{K}$ to over $200 \mu \mathrm{V} / \mathrm{K}$. We believe this is related to the order-disorder phase transition in kesterite. The thermal treatment of the samples was performed with a peak temperature of $560{ }^{\circ} \mathrm{C}$, well above the reported critical temperature of the order-disorder transition. Following a slow cooling to room temperature, $\mathrm{Cu}$ and $\mathrm{Zn}$ cations set their positions to $2 c$ and $2 d$ Wyckoff sites of $I-4$ structure, respectively, leading to a partially-ordered kesterite. The extent of the ordering process critically depends on this cooling step, and on the stoichiometry of the CZTS phase. In fact, the capability of the cations to change their position is strictly related to the thermal energy of the system and a gradual temperature reduction allows the ordered arrangement of atoms on the $\mathrm{Cu}$ and $\mathrm{Zn}$ planes. The dependence of the ordering on thermal history could explain why some authors did not observe the phase transition [6,7], whereas others did (e.g., see Devi et al., 2019; Kumar, Ansari and Khare, 2018) $[8,10]$, although they did not comment on it.
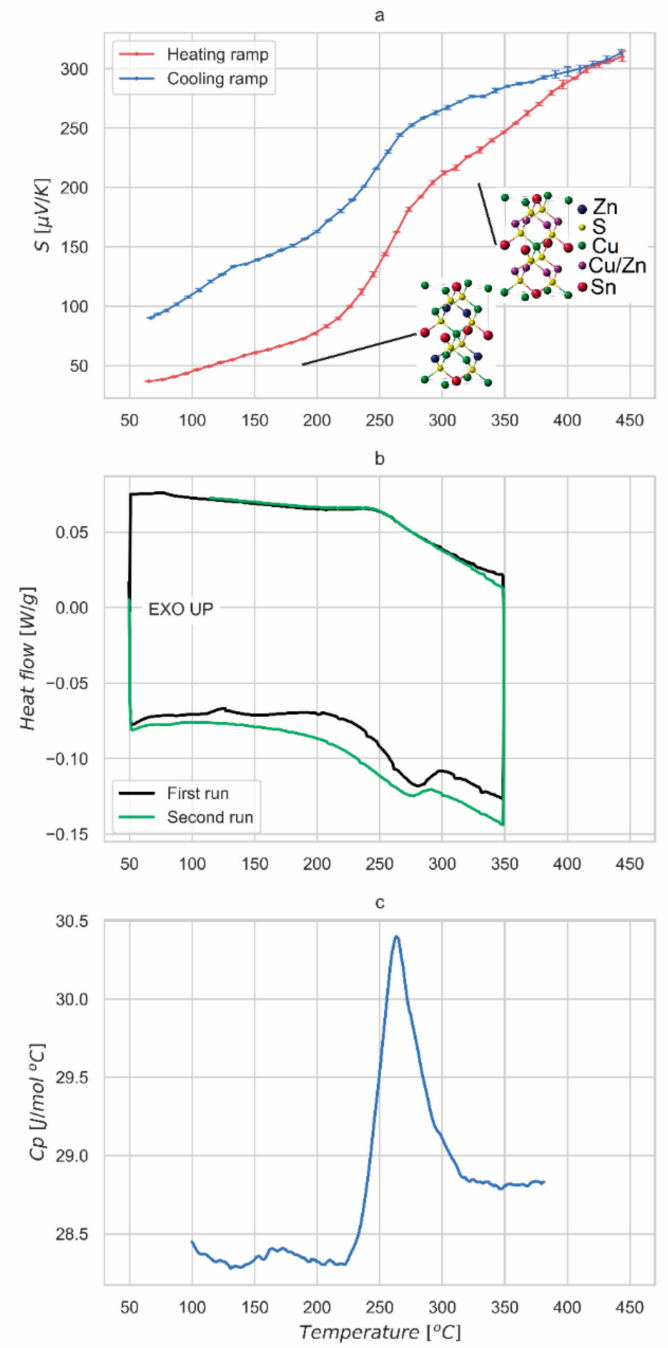

Figure 4. Seebeck measurements and thermal analyses. Absolute Seebeck coefficient measured for the slowly cooled kesterite sample in heating and cooling ramp, with insets representing the relevant crystal structures. (a). Thermal analysis (DSC) curve measured for the slowly cooled sample. First and second runs are displayed in both heating and cooling ramp. (b). A second-order phase transition is clearly visible, especially in the trend of the constant-pressure heat capacity $(C p)$, in (c). 
During Seebeck measurements, on heating phase, $\mathrm{Cu}$ and $\mathrm{Zn}$ form an increasing number of antisites, which tend to cluster in $\left(\mathrm{Cu}_{\mathrm{Zn}}+\mathrm{Zn}_{\mathrm{Cu}}\right)$ defect pairs until complete disorder is reached at the critical temperature. It is important to stress that the disordered kesterite has a different atom arrangement from stannite, although the $I-42 m$ space group is the same. On cooling, as already pointed out, the $I-4$ ordered structure is partially recovered, to an extent depending on the decreasing temperature ramp.

From the observed trend of Seebeck coefficient, we can assess the reversible character of the transition, fully developed around $260^{\circ} \mathrm{C}$ in the heating ramp: this is consistent with the order-disorder critical temperature reported by Scragg et al. [15]. The thermal hysteresis observed on the cooling ramp, lowering the critical temperature of about $20^{\circ} \mathrm{C}$, is probably related to the thermal inertia of the sample. This feature, associated to a decreasing ramp faster than the natural cooling cycle performed after sintering, can explain the difference in Seebeck coefficient observed at $50{ }^{\circ} \mathrm{C}$ between heating and cooling measurements.

It is interesting to notice how powerful the measurement of Seebeck coefficient is as a tool to study the order-disorder transition: it gives an indication of the transition temperature and of its reversible character in an easier and clearer way with respect to traditional structural characterization techniques, which are much more complex and less effective in studying this second order transition.

Thermal analysis, visible in Figure $4 \mathrm{~b}$, confirms the presence of a reversible transition. The transition has been observed between $240^{\circ} \mathrm{C}$ and $250^{\circ} \mathrm{C}$ on heating, while a low hysteresis can be observed in the reversal process during the cooling branch of the curve. The broadening of the transformation can be partially explained with the presence of secondary phases in the material. This could also explain the curve evolution between the first and the second run, probably associated with a material stabilization with thermal cycling. From the peak-shape it is confirmed to be a Landau second-order transition, matching what was previously reported for the order-disorder transition in kesterite [15].

Figure 5 shows the data collected by MDSC. The experiment allows us to separate the heat flow curve into two different contributions. One is the step change in heat capacity of the sample due to the order/disorder transition. This effect is reported in the reversible heat flow signal. The second contribution is associated with the enthalpy recovery of the system: this phenomenon is related to the kinetic of the measurement and depends mainly on the thermal diffusivity of the material and on the specimen size and shape. Such a contribution is visible as a peak in the non-reversible curve reported in Figure 5. This behavior is further proof of the nature of the transition observed in the material, which is also confirmed by the constant-pressure heat capacity $(C p)$ measurement over the same temperature interval. As shown in Figure $4 c$, a peak of $C p$ is observed at the order-disorder transition, with a shape characteristic of higher order phase transformations [39]. Cp of the high temperature (disordered) phase is higher than for the low temperature (ordered or partially-ordered) phase, demonstrating an increase in vibrational modes in the disordered phase.

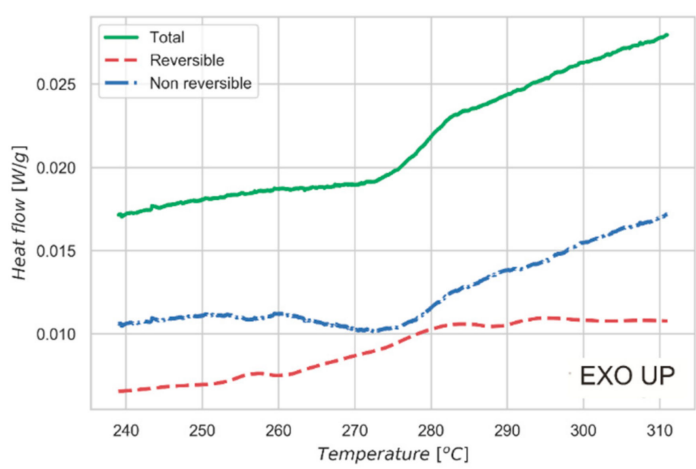

Figure 5. Modulated mode thermal analysis measurement (MDSC). MDSC is performed across the critical temperature to study the character of the order-disorder transition. MDSC allows us to separate the reversible (red) and the non-reversible (blue) contributions to the total heat flow in the sample (green). Ramp $2 \mathrm{~K} / \mathrm{min}$, modulation period $120 \mathrm{~s}$, and semi-amplitude $0.5^{\circ} \mathrm{C}$. 
To validate these observations, we measured the absolute Seebeck coefficient of a quenched sample. Quenching was performed from $560{ }^{\circ} \mathrm{C}$, well above the critical temperature, by sudden extraction of the sample from the treatment oven. Due to the fast cooling, we expect the material to be in a fully-disordered state after the treatment. The first run of the measurement of the quenched specimen (Figure 6) shows an approximately linear trend, whereas a second run, made after letting the sample to naturally cool down from the previous measurement, shows the characteristic transition shape at around $250{ }^{\circ} \mathrm{C}$. These results support the idea of a fully-disordered state for the as quenched material (first run), displaying a linear Seebeck coefficient; whereas, upon a slower cooling, kesterite partially recovers the order, displaying a sharp increase of Seebeck value during the second run, similar to the one already observed for the partially-ordered samples of Figure 4a. Further measurements of Seebeck coefficient are reported in the Supplementary Materials to show reproducibility of the analysis and the limited variability of the results.

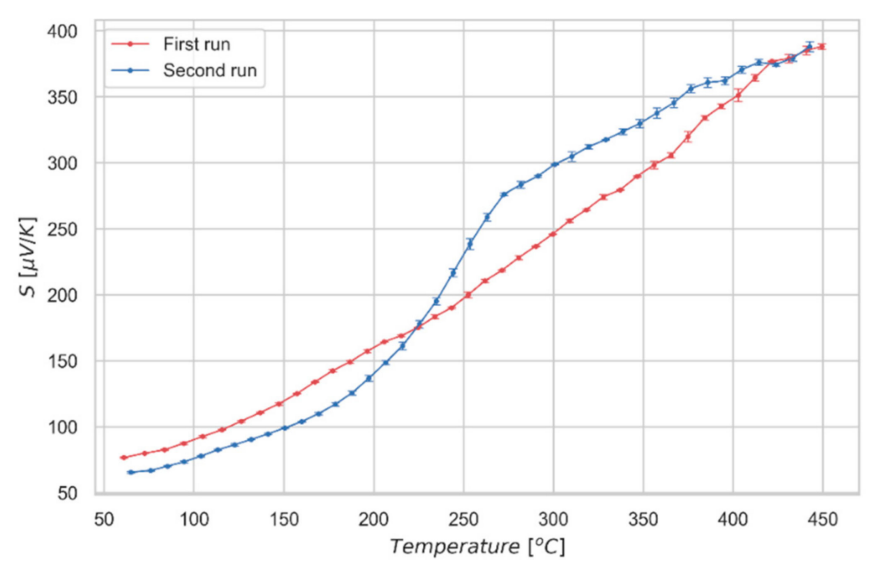

Figure 6. Absolute Seebeck coefficient measured for the quenched kesterite sample. First and second runs of the measurement are shown.

It is clear how the transition strongly affects the Seebeck of the material. In fact, in all reported cases the high-temperature Seebeck far exceeds that of the low-temperature structure. A reason for this behavior could be found in the increasing structural symmetry of the system associated with the order-disorder transition. As reported in the literature for a parental compound, the chalcopyrite $\mathrm{Cu}_{2} \mathrm{MGeSe}_{4}$, by Zeier et al. [40] and by Zhang et al. [41], the symmetry increase of the crystal structure leads to a loss of band divergence in the system, which in turn raises the density-of-states effective mass, $m_{D O S}^{*}$, of the carriers due to the increased degeneracy of the electronic bands. In the cited paper by Zeier et al. the symmetry of the system has been controlled by introducing different elements in the cell in order to obtain a pseudo-cubic structure. Here, kesterite displays a similar behavior associated with the second-order transition. The $\mathrm{Cu}-\mathrm{Zn}$ arrangement above the transition increases the symmetry due to the large number of $\left(\mathrm{Cu}_{\mathrm{Zn}}+\mathrm{Zn}_{\mathrm{Cu}}\right)$ defects promoted by the thermal energy. In fact, above the order-disorder transition, the ordered atomic arrangement, having $\mathrm{Cu}$ atoms in $2 c(0,1 / 2,1 / 4)$ positions and $\mathrm{Zn}$ in $2 d(0,1 / 2,3 / 4)$ ones, leaves the place to a statistically arranged distribution of $\mathrm{Cu}$ and $\mathrm{Zn}$ on the $4 d(0,1 / 2,1 / 4)$ positions [21]. Such disorder involves an increase in the symmetry of the system, which can result in a narrowing of the gaps between the different bands at $\Gamma$ point. This effect could lead to a band degeneracy due to the removal of the crystal field contribution, $\Delta_{C F}$, to the band spacing. As reported in the literature [42], for CZTS the spin-orbit coupling doesn't affect the band degeneracy due to the light weight of $S$ atoms.

To demonstrate the degeneracy mechanism, however, it is necessary to check in detail the band structures of the ordered and disordered states of $\mathrm{Cu}_{2} \mathrm{ZnSnS}_{4}$. Apparently, this information, so far, is only available for the ordered kesterite, close to our low temperature structure, and stannite [43,44], with same crystal structure of our disordered kesterite, but corresponding to low temperature and 
different atom arrangement. To the best of our knowledge, no information is reported in the literature on the band structure of disordered kesterite, possibly including the effects of stoichiometry fluctuations and temperature, in the range explored by our measurements of Seebeck coefficient.

The influence of the structural state of the material on its band structure, could also explain the difference observed in the Seebeck measurement performed on the rapidly cooled sample (Figure 6). In fact, the first cycle not only differs in terms of the absence of transition evidence, but also displays unexpected absolute values: considering the disordered state at low temperature kept by the quench from high temperature, values of Seebeck coefficient in the first cycle should be higher, matching the linear trend observed above the transition during the second run. However, the quenching process promotes not only a disordered structure, but also structural distortions; indeed, quenched samples tend to deform, as a clear consequence of residual stresses. The thermal cycle performed by the sample during the measurement, followed by a slow cooling at room temperature, allows the structure to recover from all the effects induced by the fast cooling. As a consequence of structural stresses, the measured Seebeck values, despite being higher in the first run, are not as high as they should for the lack of full recovery of the system. The convergence of the Seebeck values can only be observed at high temperature, where residual stress is completely relaxed.

The present work was not aimed at showing a high-performance thermoelectric material, but rather to show the structural information provided by a simple measurement of Seebeck coefficient with the support of thermal analyses. Moreover, the order-disorder transition of kesterite known in photovoltaics was presented from a different perspective, as a proof-of-concept of the possibility to achieve a higher Seebeck coefficient with increased disorder and crystal symmetry. It is however possible to estimate a figure of merit $\mathrm{zT}$ at $400{ }^{\circ} \mathrm{C}$ for our samples, ranging from 0.025 to 0.04 , comparable with the literature values for thermoelectric kesterite at the same temperature [6,7]. For this estimate we used the specific heat of Figure 4 and the published data of electrical resistivity and thermal diffusivity [31]. The latter were recently measured on a kesterite sample obtained with the same process, apart from a faster cooling down process after the thermal treatment; the zT value should be unaffected in any case by the cooling velocity, since the sample at $400{ }^{\circ} \mathrm{C}$ is in a complete disordered state. More extensive measurements and developments to improve electrical resistivity and thermal diffusivity of the studied system are part of the ongoing research work.

\section{Conclusions}

The present work demonstrates the effect of the order-disorder transition of kesterite on the Seebeck coefficient. This transition, known as a detrimental effect for the performance of solar cell materials, turns out to positively affect the thermopower. $C p$ measurement and thermal analyses, including modulated mode measurements (MDSC) to decouple the reversible and non-reversible components of heat flow, confirm what was previously reported in the literature about the character of the transition [14]: it is a second-order Landau phase transition, completely developed at $260{ }^{\circ} \mathrm{C}$ and fully reversible upon slow cooling. The Seebeck curve presents a sharp increase of more than $100 \mu \mathrm{V} / \mathrm{K}$ at the transition temperature, from around $80 \mu \mathrm{V} / \mathrm{K}$ at $210^{\circ} \mathrm{C}$ to over $200 \mu \mathrm{V} / \mathrm{K}$ at $290{ }^{\circ} \mathrm{C}$. The effect of $\mathrm{Cu}-\mathrm{Zn}$ disorder has been further assessed by measuring the Seebeck coefficient of a quenched sample (in disordered state, I-42m crystal structure) and observing that it follows a linear trend, while after slow cooling (partially recovers ordered $I-4$ crystal structure) displays a sharp increase due to the transition. Measuring the Seebeck coefficient also proved to be a simple and efficient way to observe the transition, which is difficult to spot using traditional structural characterization techniques [20,21]. What shown in this paper leads us to think that the order-disorder transition of kesterite and, in a wider sense, the evolution towards a more symmetrical crystalline structure and the cationic disorder, can lead to an increase in band degeneration and to the consequent improvement of the Seebeck coefficient. This indicates some promising study directions for further improvement of thermoelectric performance. 
Supplementary Materials: The following are available online at http://www.mdpi.com/2079-4991/9/5/762/s1.

Author Contributions: Conceptualization, P.S. and E.I.; methodology, P.S. and C.F.; XRD data modeling, E.I. and P.S.; data interpretation, C.F., P.S. and E.I.; experimental investigation, E.I. and C.F.; data curation, E.I. and C.F.; writing—original draft preparation, E.I.; writing—review and editing, P.S., C.F., N.M.P.; supervision, P.S., N.M.P.

Funding: This research was funded by the Autonomous Province of Trento, within the framework of the programmatic Energy Action 2015-2017. N.M.P. was supported by the European Commission under the Graphene Flagship Core 2 grant No. 785219 (WP14 "Composites") and FET Proactive "Neurofibres" grant No. 732344 as well as by the Italian Ministry of Education, University and Research (MIUR), under the "Departments of Excellence" grant L. 232/2016 and ARS01-01384-PROSCAN grant.

Acknowledgments: The authors would like to acknowledge the help of G. Ischia of Electron Microscopy Facility of the Laboratory of Micro \& Structural Analysis (Department of Industrial Engineering, Trento) and Corrado Tomasi of CNR, Genova Unit, for useful discussions.

Conflicts of Interest: The authors declare no conflict of interest.

\section{References}

1. Seebeck, T.J. Ueber die magnetische Polarisation der Metalle und Erze durch Temperaturdifferenz. [Magnetic polarization of metals and ores by temperature differences]. Abhandlungen der K. Akad. der Wissenschaften zu Berlin 1826, 82, 265-373. (In German)

2. Peltier, J.C.H. Nouvelles expériences sur la caloricité des courants électrique. [New experiments on the heat effects of electric currents]. Ann. Chim. Phys. 1834, 56, 371-386. (In French)

3. Rowe, D.M. Thermoelectrics and Its Energy Harvesting; CRC PRESS: Boca Raton, FL, USA, 2012; ISBN 9781439874707 (hardback v.1) 9781439874721 (hardback v.2).

4. Zeier, W.G.; Snyder, G.J.; Zevalkink, A.; Gibbs, Z.M.; Hautier, G.; Kanatzidis, M.G. Thinking Like a Chemist: Intuition in Thermoelectric Materials. Angew. Chem. Int. Ed. 2016, 55, 6826-6841. [CrossRef]

5. Zeier, W.G. New tricks for optimizing thermoelectric materials. Curr. Opin. Green Sustain. Chem. 2017, 4, 23-28. [CrossRef]

6. Liu, M.L.; Huang, F.Q.; Chen, L.D.; Chen, I.W. A wide-band-gap p-type thermoelectric material based on quaternary chalcogenides of $\mathrm{Cu} 2 \mathrm{ZnSnQ4}(\mathrm{Q}=\mathrm{S}, \mathrm{Se})$. Appl. Phys. Lett. 2009, 94. [CrossRef]

7. Yang, H.; Jauregui, L.A.; Zhang, G.; Chen, Y.P.; Wu, Y. Nontoxic and abundant copper zinc tin sulfide nanocrystals for potential high-temperature thermoelectric energy harvesting. Nano Lett. 2012, 12, 540-545. [CrossRef] [PubMed]

8. Kumar, S.; Ansari, M.Z.; Khare, N. Influence of compactness and formation of metallic secondary phase on the thermoelectric properties of Cu2ZnSnS4 thin films. Thin Solid Films 2018, 645, 300-304. [CrossRef]

9. Sharma, S.D.; Neeleshwar, S. Thermoelectric Properties of hot pressed CZTS micro spheres synthetized by microwave method. MRS Adv. 2018, 3, 1373-1378. [CrossRef]

10. Sharma, S.D.; Khasimsaheb, B.; Chen, Y.Y.; Neeleshwar, S. Enhanced thermoelectric performance of Cu 2 ZnSnS 4 (CZTS) by incorporating Ag nanoparticles. Ceram. Int. 2019, 45, 2060-2068. [CrossRef]

11. Katagiri, H.; Jimbo, K.; Yamada, S.; Kamimura, T.; Maw, W.S.; Fukano, T.; Ito, T.; Motohiro, T. Enhanced conversion efficiencies of Cu2ZnSnS4-based thin film solar cells by using preferential etching technique. Appl. Phys. Express 2008, 1. [CrossRef]

12. Mitzi, D.B.; Gunawan, O.; Todorov, T.K.; Barkhouse, D.A.R. Prospects and performance limitations for Cu-Zn-Sn-S-Se photovoltaic technology. Philos. Trans. R. Soc. A 2013, 371, 20110432. [CrossRef] [PubMed]

13. Adachi, S. Introduction, 1st ed.; John Wiley and Sons, Ltd.: Chichester West Sussex, UK, 2015; ISBN 9781119052814.

14. Scragg, J.J.S.; Choubrac, L.; Lafond, A.; Ericson, T.; Platzer-Björkman, C. A low-temperature order-disorder transition in Cu2ZnSnS4 thin films. Appl. Phys. Lett. 2014, 104, 041911. [CrossRef]

15. Scragg, J.J.S.; Larsen, J.K.; Kumar, M.; Persson, C.; Sendler, J.; Siebentritt, S. Cu-Zn disorder and band gap fluctuations in Cu2ZnSn(S,Se)4: Theoretical and experimental investigations. Phys. Satus Solidi B 2016, 253, 247-254. [CrossRef]

16. Chen, S.; Gong, X.G.; Walsh, A.; Wei, S. Defect physics of the kesterite thin-film solar cell absorber. Appl. Phys. Lett. 2010, 96, 021902. [CrossRef]

17. Rudisch, K.; Davydova, A.; Platzer-björkman, C. The effect of stoichiometry on Cu-Zn ordering kinetics in Cu2ZnSnS4 thin films. J. Appl. Phys. 2018, 123, 161558. [CrossRef] 
18. Valentini, M.; Malerba, C.; Menchini, F.; Tedeschi, D.; Polimeni, A.; Capizzi, M.; Mittiga, A. Effect of the order-disorder transition on the optical properties of Cu2ZnSnS4. Appl. Phys. Lett. 2016, 108, 211909. [CrossRef]

19. Malerba, C.; Valentini, M.; Mittiga, A. Cation Disorder in Cu2ZnSnS4 Thin Films: Effect on Solar Cell Performances. Sol. RRL 2017, 1, 1700101. [CrossRef]

20. Schorr, S.; Gonzalez-Aviles, G. In-situ investigation of the structural phase transition in kesterite. Phys. Status Solidi Appl. Mater. Sci. 2009, 206, 1054-1058. [CrossRef]

21. Ritscher, A.; Hoelzel, M.; Lerch, M. The Order-Disorder Transition in Cu2ZnSnS4-A Neutron Scattering Investigation. J. Solid State Chem. 2016, 238, 68-73. [CrossRef]

22. Paris, M.; Lafond, A.; Guillot-deudon, C. Solid-State NMR and Raman Spectroscopy To Address the Local Structure of Defects and the Tricky Issue of the $\mathrm{Cu} / \mathrm{Zn}$ Disorder in Cu-Poor, Zn-Rich CZTS Materials. Inorg. Chem. 2014, 53, 8646-8653. [CrossRef] [PubMed]

23. Rey, G.; Redinger, A.; Weiss, T.P.; Guennou, M. The band gap of Cu2ZnSnSe4: Effect of order-disorder. Appl. Phys. Lett. 2014, 105, 112106. [CrossRef]

24. Dimitrievska, M.; Saucedo, E.; Jawhari, T.; Pérez-Rodríguez, A.; Fontané, X. Multiwavelength excitation Raman scattering study of polycrystalline kesterite Cu2ZnSnS4 thin films. Appl. Phys. Lett. 2014, 104, 021901. [CrossRef]

25. Stone, K.H.; Christensen, S.T.; Harvey, S.P.; Teeter, G.; Repins, I.L.; Repins, I.L.; Toney, M.F. Quantifying point defects in $\mathrm{Cu} 2 \mathrm{ZnSn}(\mathrm{S}, \mathrm{Se}) 4$ thin films using resonant x-ray diffraction. Appl. Phys. Lett. 2016, 109, 161901. [CrossRef]

26. Chen, S.; Walsh, A.; Gong, X.; Wei, S. Classification of Lattice Defects in the Kesterite Cu2ZnSnS4 and Cu2ZnSnSe4 Earth-Abundant Solar Cell Absorbers. Adv. Mater. 2013, 25, 1522-1539. [CrossRef] [PubMed]

27. Gabka, G.; Bujak, P.; Gryszel, M.; Ostrowski, A.; Malinowska, K.; Zukowska, G.Z.; Agnese, F.; Pron, A.; Reiss, P. Synthesis and surface chemistry of high quality wurtzite and kesterite $\mathrm{Cu} 2 \mathrm{ZnSnS4}$ nanocrystals using tin(II) 2-ethylhexanoate as a new tin source. Chem. Commun. 2015, 51, 12985-12988. [CrossRef]

28. Ataollahi, N.; Malerba, C.; Ciancio, R.; Edla, R.; Scardi, P.; Cappelletto, E.; Di Maggio, R. Control of composition and grain growth in $\mathrm{Cu} 2 \mathrm{ZnSnS4}$ thin films from nanoparticle inks. Thin Solid Films 2019, 674, 12-21. [CrossRef]

29. Ricardo, C.L.A.; Su, M.S.; Müller, M.; Scardi, P. Production of Cu2(Zn,Fe)SnS4 powders for thin film solar cell by high energy ball milling. J. Power Sources 2013, 230, 70-75. [CrossRef]

30. Broseghini, M.; Gelisio, L.; D'Incau, M.; Azanza Ricardo, C.L.; Pugno, N.M.; Scardi, P. Modeling of the planetary ball-milling process: The case study of ceramic powders. J. Eur. Ceram. Soc. 2016, 36, 2205-2212. [CrossRef]

31. Isotta, E.; Pugno, N.M.; Scardi, P. Nanostructured kesterite (Cu2ZnSnS4) for applications in thermoelectric devices. Powder Diffr. 2019, 1-6. [CrossRef]

32. Coelho, A.A. TOPAS and TOPAS-Academic: An optimization program integrating computer algebra and crystallographic objects written in C++. J. Appl. Crystallogr. 2018, 51, 210-218. [CrossRef]

33. [Database] ICDD. PDF-4+ 2019 (Database); Kabekkodu, S., Ed.; International Centre for Diffraction Data: Newtown Square, PA, USA, 2019.

34. Matsushita, H.; Ichikawa, T.; Katsui, A. Structural, thermodynamical and optical properties of Cu 2-II-IV-VI4 quaternary compounds. J. Mater. Sci. 2005, 40, 2003-2005. [CrossRef]

35. Guen, L.; Glaunsinger, W.S. Electrical, magnetic, and EPR studies of the quaternary chalcogenides Cu2AIIBIVX4 prepared by iodine transport. J. Solid State Chem. 1980, 35, 10-21. [CrossRef]

36. Scardi, P.; Leoni, M. Whole powder pattern modeling. Acta Crystallogr. Sect. A Found. Crystallogr. 2002, 58, 190-200. [CrossRef]

37. Scardi, P.; Azanza Ricardo, C.L.; Perez-Demydenko, C.; Coelho, A.A. Whole powder pattern modeling macros for TOPAS. J. Appl. Crystallogr. 2018, 51, 1752-1765. [CrossRef]

38. Scardi, P. Microstructural Properties: Lattice Defects and Domain Size Effects. In Powder Diffraction: Theory and Practice; Royal Society of Chemistry: Cambridge, UK, 2008; pp. 378-417.

39. Mott, N.F.; Jones, H. The Theory of the Properties of Metals and Alloys. J. Chem. Educ. 1936, 14, 99.

40. Zeier, W.G.; Zhu, H.; Gibbs, Z.M.; Ceder, G.; Tremel, W.; Snyder, G.J. Band convergence in the non-cubic chalcopyrite compounds Cu2MGeSe4. J. Mater. Chem. C 2014, 2, 10189-10194. [CrossRef] 
41. Zhang, Q.; Song, Q.; Wang, X.; Sun, J.; Zhu, Q.; Dahal, K.; Lin, X.; Cao, F.; Zhou, J.; Chen, S.; et al. Deep defect level engineering: A strategy of optimizing the carrier concentration for high thermoelectric performance. Energy Environ. Sci. 2018, 11, 933-940. [CrossRef]

42. Wang, H.; Pei, Y.; Lalonde, A.D.; Snyder, G.J. Thermoelectric Nanomaterials; Springer: Berlin, Germany, 2013; Volume 182, ISBN 978-3-642-37536-1.

43. Botti, S.; Kammerlander, D.; Marques, M.A.L. Band structures of Cu2 ZnSnS4 and Cu2 ZnSnSe4 from many-body methods. Appl. Phys. Lett. 2011, 98, 241915. [CrossRef]

44. Wang, B.; Xiang, H.; Nakayama, T.; Zhou, J.; Li, B. Theoretical investigation on thermoelectric properties of Cu-based chalcopyrite compounds. Phys. Rev. B 2017, 95, 035201. [CrossRef]

(C) 2019 by the authors. Licensee MDPI, Basel, Switzerland. This article is an open access article distributed under the terms and conditions of the Creative Commons Attribution (CC BY) license (http://creativecommons.org/licenses/by/4.0/). 